Supporting information

\title{
Elemental sulfur-stabilized liquid marbles: \\ Properties and applications
}

Sajedeh Mahmoudi Salehabad, Saeid Azizian*

Department of Physical Chemistry, Faculty of Chemistry, Bu-Ali Sina University, Hamedan 65167, Iran

\section{Corresponding Author}

*E-mail: sazizian@basu.ac.ir. 


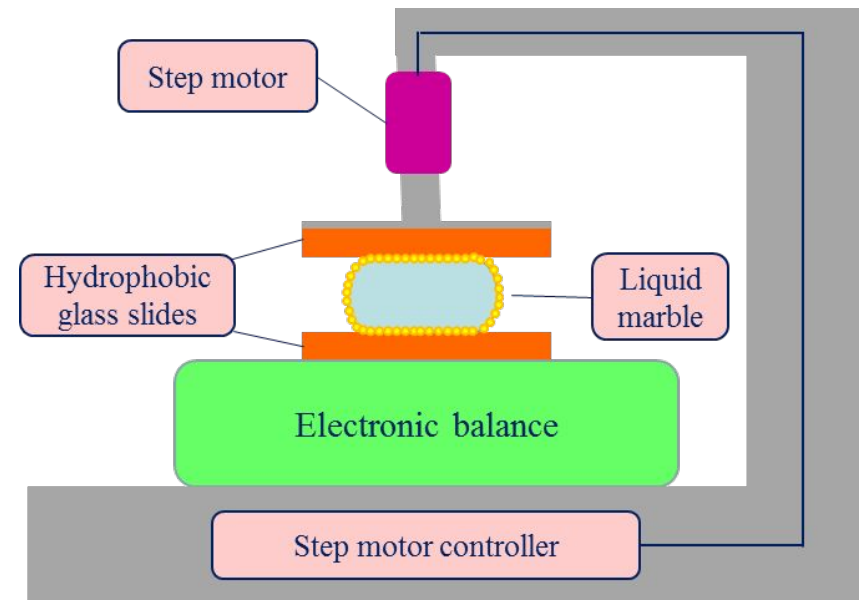

Figure S1. Schematic of compression test setup. A liquid marble is compressed between hydrophobic glass slides at a constant speed. An electronic balance is used to monitor the compression force.
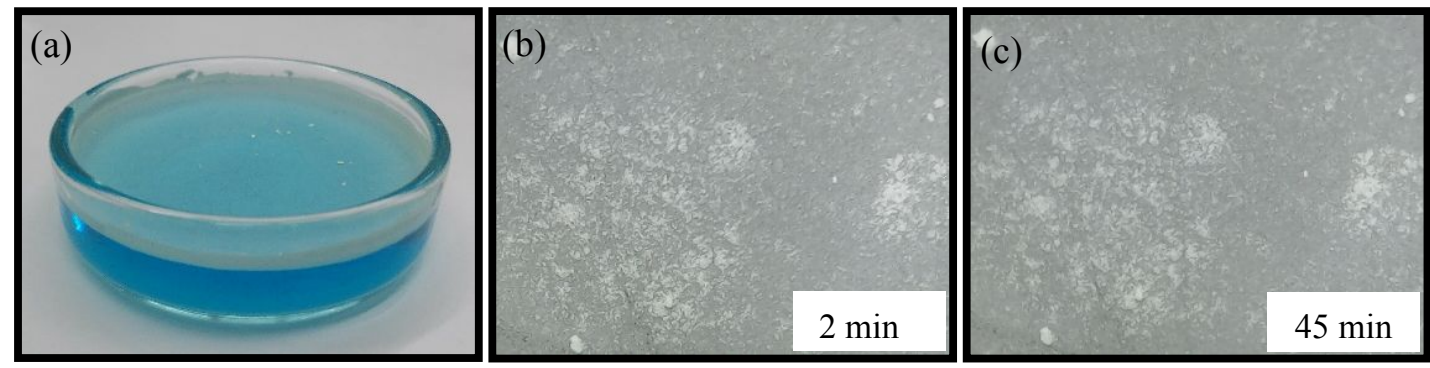

Figure S2. (a) The sulfur gel layer formed on planar water surface (dyed with methylene blue) in the Petri dish. (b, c) Top-view images of gel layer formed on planar water surfaces $(\mathrm{pH}=6)$ at different time.
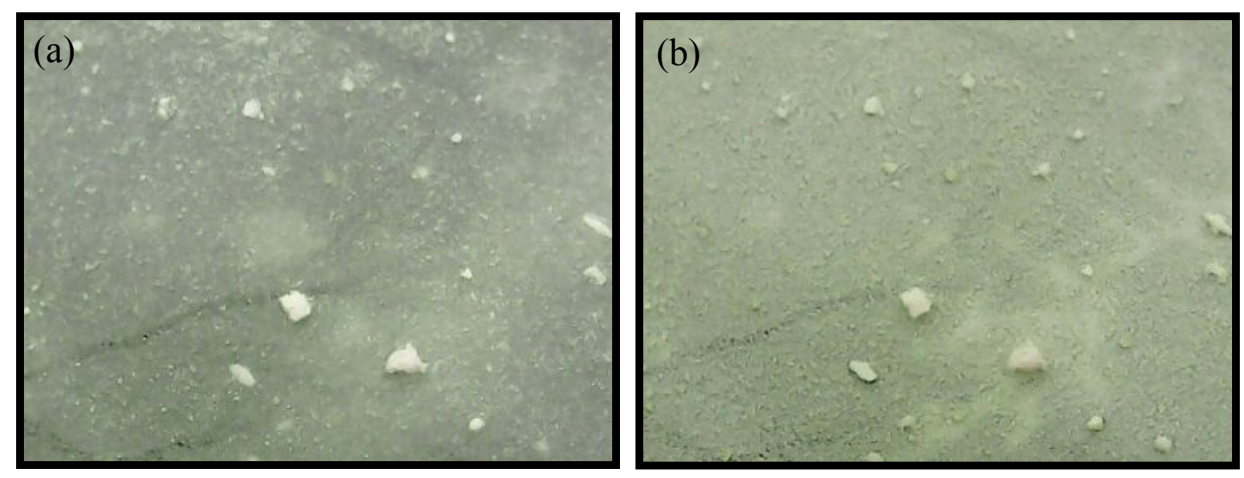

Figure S3. Deposited sulfur gel layer on glass slide (a) fresh sample and (b) 60 min after deposition. 


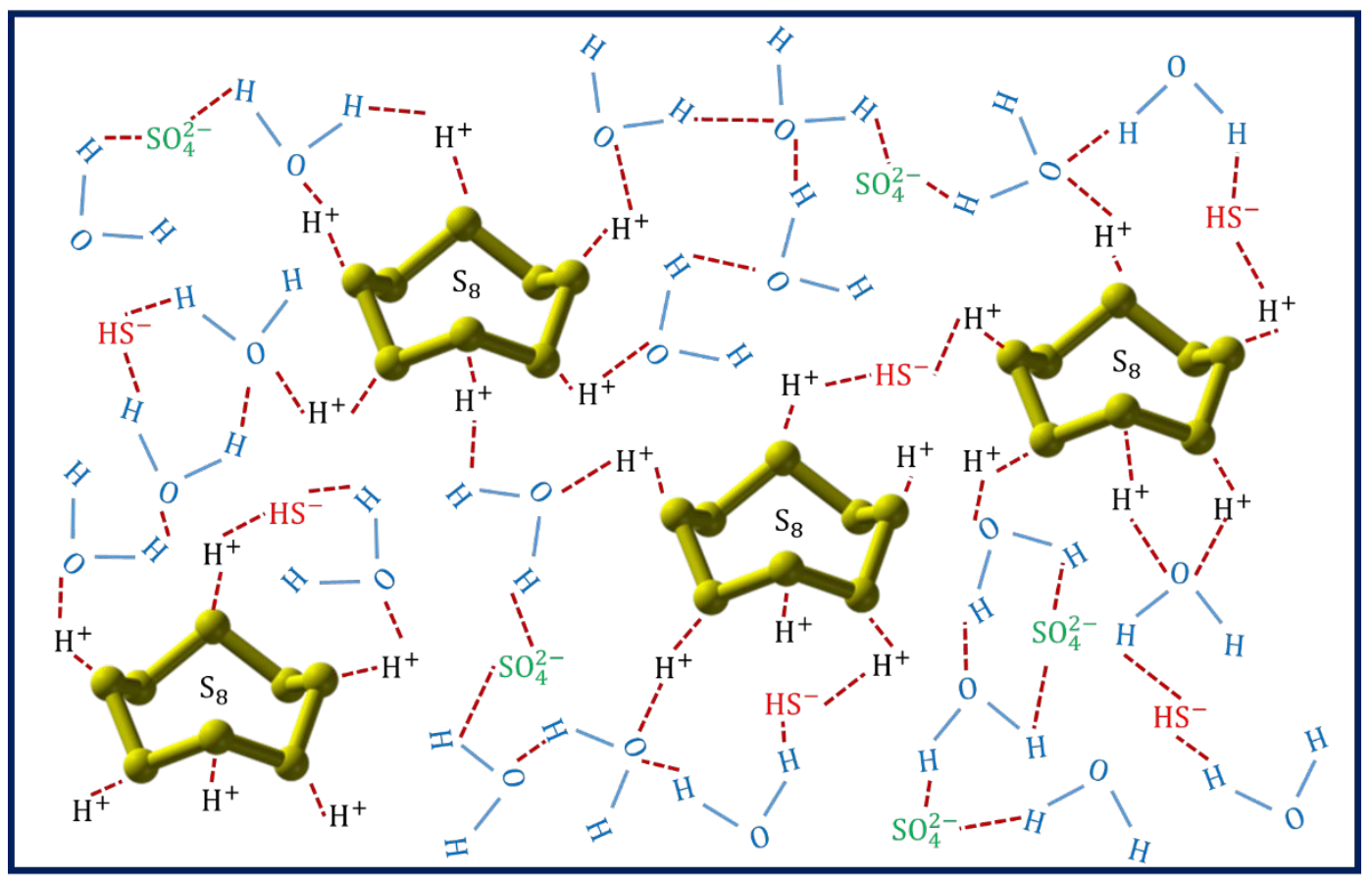

Figure S4. Proposed schematic of gel layer constructed on sulfur-stabilized liquid marbles surface.

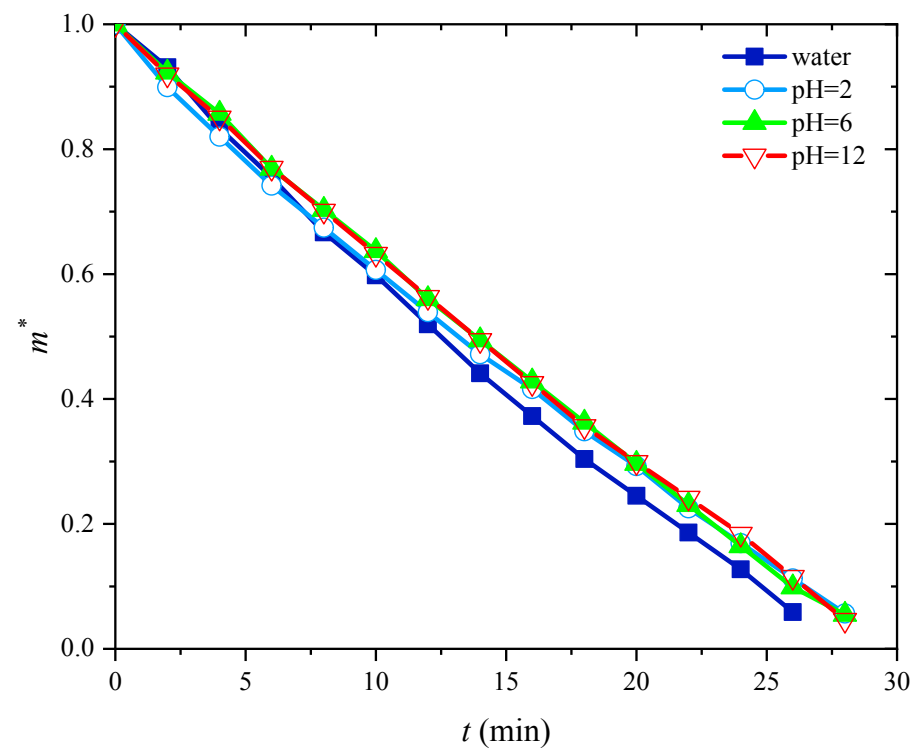

Figure S5. The evaporation rate of $5 \mu \mathrm{L}$ liquid marbles fabricated using aqueous solutions with different $\mathrm{pH}$ values at $26^{\circ} \mathrm{C}$ temperature and $30 \%$ relative humidity. 


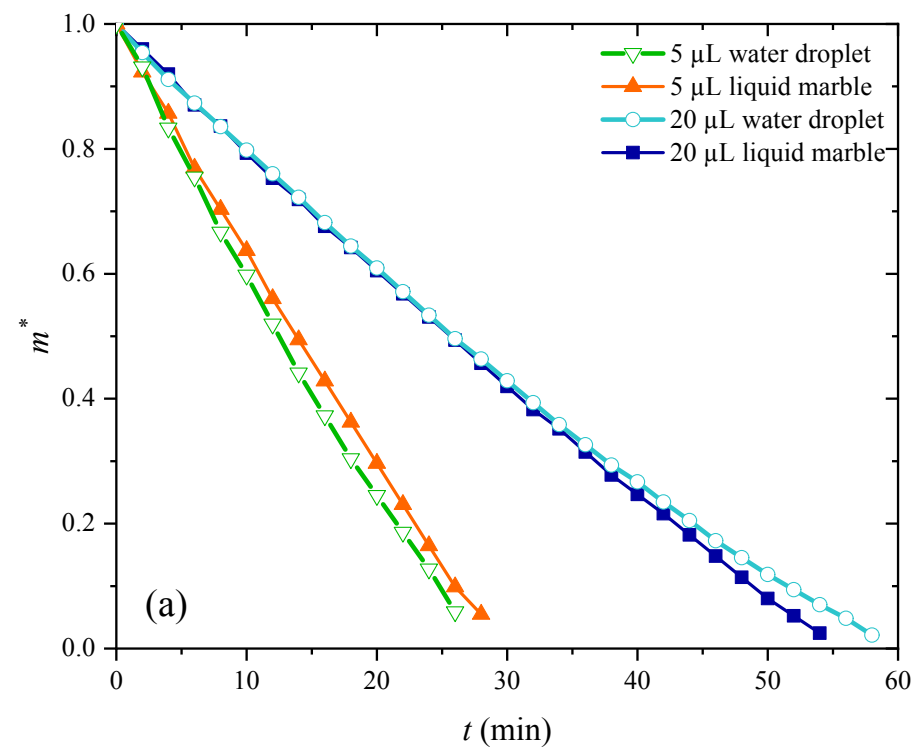

Figure S6. The evaporation rate of liquid marbles fabricated using deionized water with different volumes at $26^{\circ} \mathrm{C}$ temperature and $30 \%$ relative humidity.

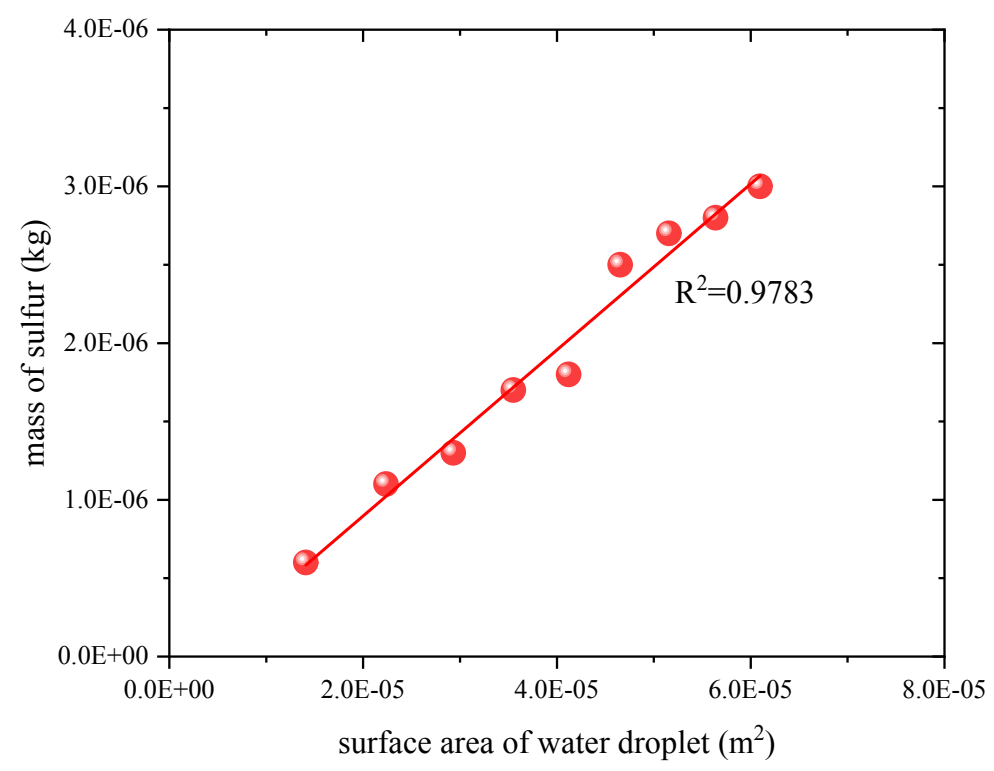

Figure S7. Change in mass of sulfur particles encapsulating the liquid marble by changing in surface area of water droplet with 5-45 $\mu \mathrm{L}$ volumes. 

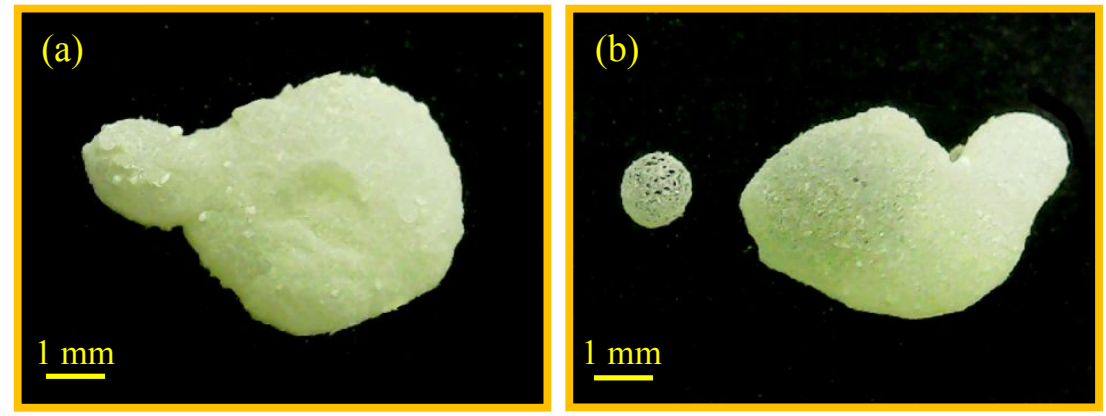

Figure S8. The $20 \mu \mathrm{L}$ deionized liquid marbles $(\mathrm{pH}=6)$ prepared by $5 \mathrm{~s}$ rolling on sulfur powder after dropping on solid substrate from (a) $5 \mathrm{~cm}$ and (b) $9 \mathrm{~cm}$ heights.
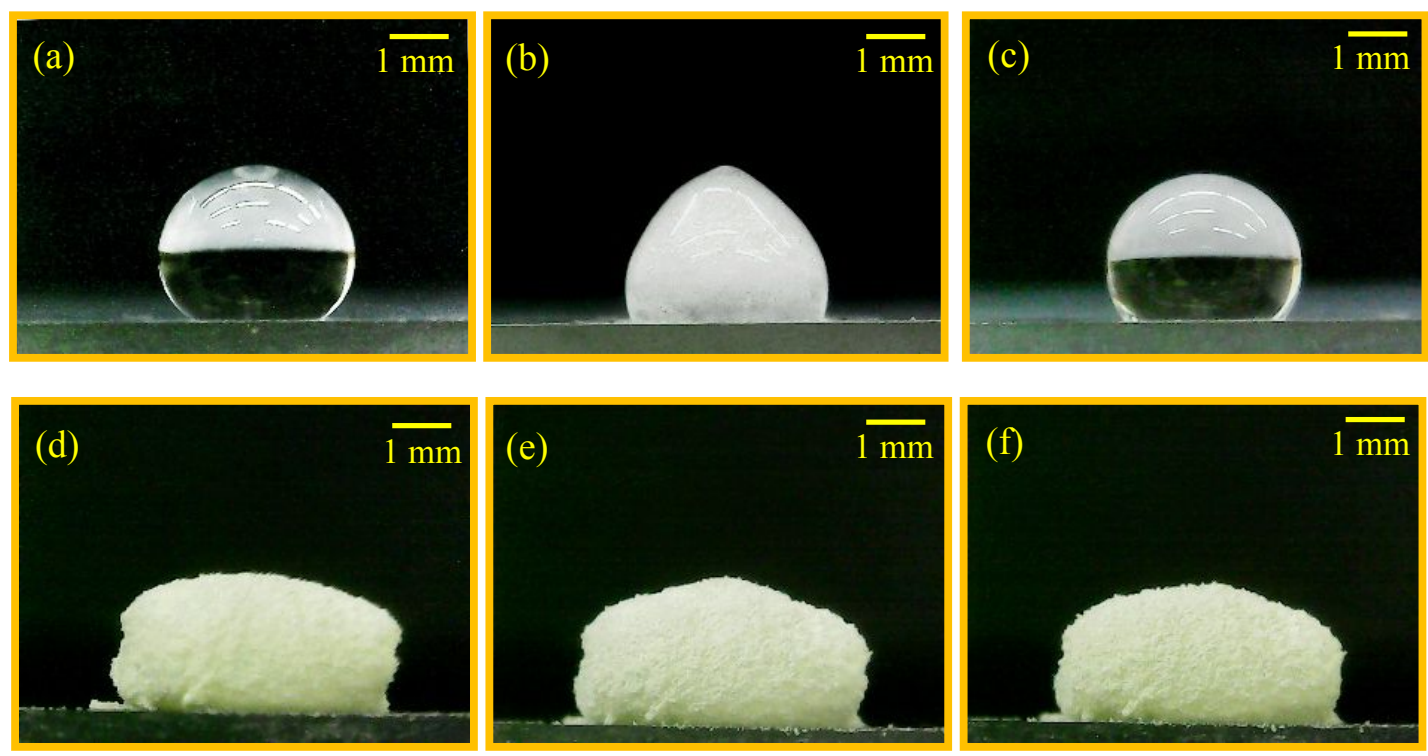

Figure S9. The images of $15 \mu \mathrm{L}$ (a-c) bare water droplet and (d-f) sulfur liquid marble with $\mathrm{pH}=6$ after 40 s rolling on sulfur powder: $(a, d)$ fresh sample, $(b, e)$ freezed sample and $(c, f)$ re-melted sample.

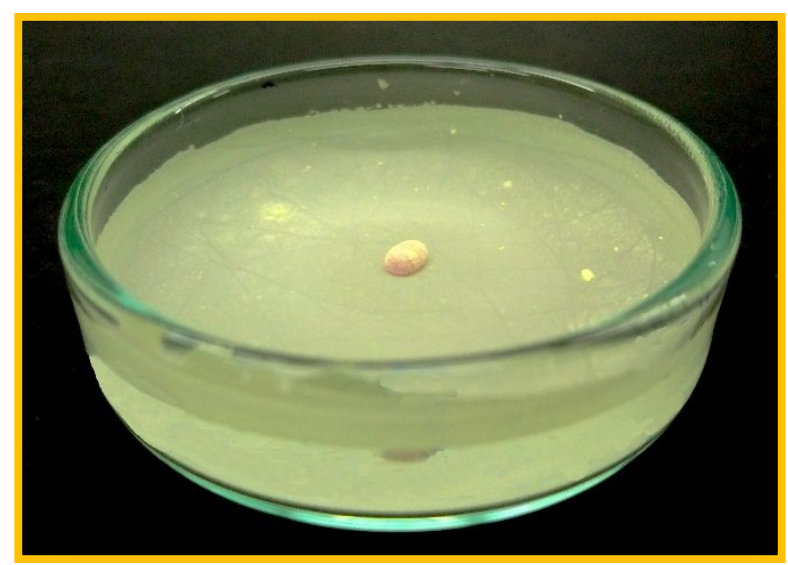

Figure S10. A $20 \mu \mathrm{L}$ floating sulfur stabilized liquid marble on top of sulfur hydrogel layer formed on water surface. 

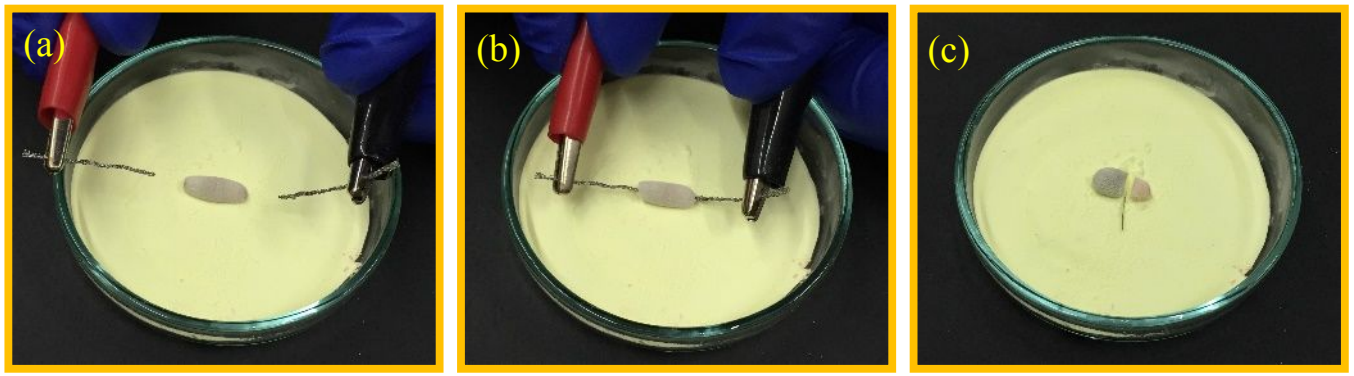

Figure S11. An electrochemical microcell designed by $60 \mu \mathrm{L}$ sulfur-stabilized liquid marble fabricated using deionized water dyed with Congo red: (a) before electrodes insertion, (b) one minute after electrodes insertion and (c) two remained small marbles after cutting the liquid marble by a scalpel.
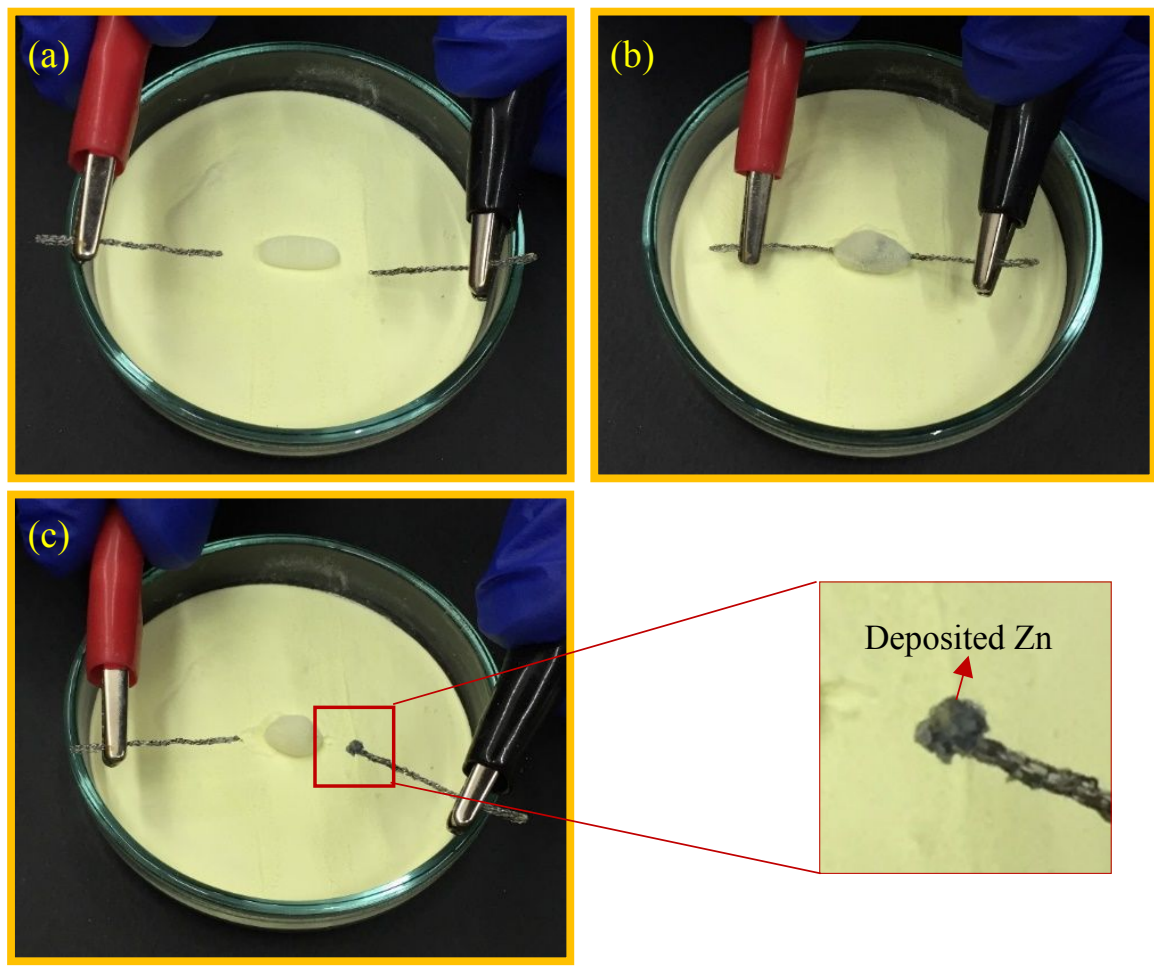

Figure S12. An electrochemical microcell designed by $60 \mu \mathrm{L}$ sulfur-stabilized liquid marble fabricated using $0.1 \mathrm{M}$ zinc acetate solution: (a) before electrodes insertion, (b) one minute after electrodes insertion and (c) deposited zinc on steel wire after one minute. 
Table S1. The evaporation rates for sulfur stabilized liquid marbles with $5 \mu \mathrm{L}$ of aqueous solution at different $\mathrm{pH}$ values compared with $5 \mu \mathrm{L}$ uncoated deionized water droplet with $\mathrm{pH}=6$.

\begin{tabular}{|l|c|c|c|c|}
\hline & Water droplet & $\mathrm{pH}=2$ & $\mathrm{pH}=6$ & $\mathrm{pH}=12$ \\
\hline$\frac{d m^{*}}{d t}\left(\mathrm{~min}^{-1}\right)$ & $-3.6 \times 10^{-2}$ & $-3.3 \times 10^{-2}$ & $-3.4 \times 10^{-2}$ & $-3.3 \times 10^{-2}$ \\
& & & & \\
\hline
\end{tabular}

Table S2. Quantitative gravimetric data for sulfur particles surrounding the liquid droplets.

\begin{tabular}{|c|c|}
\hline Sulfur particles mass per droplet surface area & $5.30 \times 10^{-2} \mathrm{~kg} \mathrm{~m}^{-2}$ \\
\hline Mean sulfur particles diameter & $9.12 \times 10^{-6} \mathrm{~m}$ \\
\hline Sulfur single particle volume & $3.17 \times 10^{-15} \mathrm{~m}^{3}$ \\
\hline Sulfur particle density & $2015 \mathrm{~kg} \mathrm{~m}^{-3}$ \\
\hline Sulfur single particle mass & $6.40 \times 10^{-12} \mathrm{~kg}$ \\
\hline $\begin{array}{l}\text { Number of sulfur particles at the air-water } \\
\text { interface of a liquid marble }\end{array}$ & $1.24 \times 10^{10}$ particles per $\mathrm{m}^{2}$ \\
\hline Sulfur particle surface area & $2.61 \times 10^{-10} \mathrm{~m}^{2}$ \\
\hline $\begin{array}{c}\text { Number of sulfur particles at the } 1 \mathrm{~m}^{2} \text { planar air- } \\
\text { water interface }\end{array}$ & $3.83 \times 10^{9}$ particles per $\mathrm{m}^{2}$ \\
\hline Number of sulfur monolayers on a liquid marble & $\sim 3$ monolayers \\
\hline
\end{tabular}


Table S3. Comparison of properties of different deformable liquid marbles.

\begin{tabular}{|c|c|c|c|c|c|c|c|c|}
\hline Liquid marble & Components & $\begin{array}{l}\text { Preparation } \\
\text { method of } \\
\text { liquid } \\
\text { marble }\end{array}$ & $\begin{array}{c}\text { Reason of } \\
\text { deformability }\end{array}$ & $\begin{array}{l}\text { Shape } \\
\text { stability } \\
\text { after } \\
\text { removing } \\
\text { external } \\
\text { force }\end{array}$ & $\begin{array}{l}\text { Stability at } \\
\text { different } \mathrm{pH}\end{array}$ & $\begin{array}{l}\text { Relative price } \\
\text { (solid } \\
\text { particles/sulfur) }\end{array}$ & Applications & Ref. \\
\hline $\begin{array}{l}\text { Modified } \mathrm{SiO}_{2} \\
\text { liquid marble }\end{array}$ & $\begin{array}{c}\mathrm{SiO}_{2}+ \\
\text { dimethyldichlorosilane }\end{array}$ & $\begin{array}{l}\text { Suction of } \\
\text { inside } \\
\text { water by } \\
95 \%\end{array}$ & $\begin{array}{l}\text { Increasing } \\
\text { particle } \\
\text { surface } \\
\text { density and } \\
\text { low water } \\
\text { content }\end{array}$ & Stable & $\begin{array}{l}\text { Probably low } \\
\text { stability at } \\
\text { high pH }\end{array}$ & $>50$ & Not reported & 23 \\
\hline $\begin{array}{l}\text { Superhydrophobic } \\
\mathrm{SiO}_{2} \text { liquid marble }\end{array}$ & $\begin{array}{c}\mathrm{SiO}_{2}+ \\
\text { hexamethyldisilazane }\end{array}$ & $\begin{array}{l}\text { Vertical } \\
\text { squeezing }\end{array}$ & $\begin{array}{l}\text { Nanoparticles } \\
\text { jamming by } \\
\text { squeezing } \\
\text { force }\end{array}$ & Stable & Not reported & $>50$ & $\begin{array}{l}\text { Liquid leases, Channel-like } \\
\text { containers }\end{array}$ & 27 \\
\hline $\begin{array}{l}\text { Stearic acid liquid } \\
\text { marble }\end{array}$ & $\mathrm{C}_{18} \mathrm{H}_{36} \mathrm{O}_{2}$ & $\begin{array}{l}\text { Back and } \\
\text { forth } \\
\text { rolling }\end{array}$ & $\begin{array}{l}\text { Gel layer } \\
\text { formation }\end{array}$ & Stable & $\begin{array}{c}\text { Low } \\
\text { deformability } \\
\text { at high } \mathrm{pH}\end{array}$ & $>2$ & $\begin{array}{l}\text { Electrochemical microcell, } \\
\text { Separable container }\end{array}$ & 32 \\
\hline PTFE & Polytetrafluoroethylene & $\begin{array}{l}\text { Actuation } \\
\text { with } \\
\text { electric } \\
\text { field }\end{array}$ & $\begin{array}{l}\text { Dipole-dipole } \\
\text { interaction } \\
\text { with electric } \\
\text { field }\end{array}$ & $\begin{array}{l}\text { Non- } \\
\text { stable }\end{array}$ & Not reported & $>30$ & Not reported & $*$ \\
\hline Sulfur liquid marble & $\mathrm{S}_{8}$ & $\begin{array}{l}\text { Back and } \\
\text { forth } \\
\text { rolling }\end{array}$ & $\begin{array}{l}\text { Gel layer } \\
\text { formation }\end{array}$ & Stable & $\begin{array}{l}\text { Stable and } \\
\text { deformable at } \\
\text { whole } \mathrm{pH} \\
\text { values }\end{array}$ & 1 & $\begin{array}{l}\text { Photocatalytic microreactor, } \\
\text { Electrochemical microcell, } \\
\text { Water in oil microreactor, } \\
\text { Separable container }\end{array}$ & $\begin{array}{l}\text { This } \\
\text { work }\end{array}$ \\
\hline
\end{tabular}

* Bormashenko, E., et al., 2012. Composite Non-Stick Droplets and Their Actuation with Electric Field. Applied Physics Letters, $100(15)$, pp.151601. 